\title{
WORKING GROUP FOR THE WORLDWIDE DEVELOPMENT OF ASTRONOMY
}

\author{
(GROUPE DE TRAVAIL POUR LE DEVELOPPEMENT \\ MONDIAL DE L'ASTRONOMIE)
}

Report of Meeting held on Saturday, 20th August, 1994.

\author{
Chairman: A.H. Batten
}

Some forty to fifty people attended the open meeting of the Group held to discuss the problems of astronomy in Africa. Representatives of all five countries on that continent in which IAU members reside were able to make presentations. Some others with particular interests related to African astronomy also contributed. The speakers were: L.I.Onuora (Nigeria), R.Sadat (Algeria), K.Chamcham (Morocco), A.M.I.Osman (Saudi Arabia), F.R.Querci (France), A.Boksenberg (U.K.) and D.L.Crawford (U.S.A.). A fuller account of the proceedings, together with summaries of the talks and discussion, will be found in Highlights of Astronomy.

Meeting of Tuesday, 23rd August, 1994

Chairman: A.H.Batten

Attendance at this meeting was restricted by invitation. About a dozen IAU members met to discuss with the Assistant General Secretary the particular problems of Astronomy in Viet Nam. A Vietnamese astronomer was among those attending. Discussion focussed on the October 1995 eclipse of the Sun, which will be total in the southern part of Viet Nam. It was planned to use this occasion to launch an IAU initiative in that country. It was proposed to hold a small meeting in conjunction with the eclipse, which might possibly also serve to initiate the new Teaching for Astronomy Development programme. Ways and means of encouraging cooperation between Vietnamese astronomers and colleagues in neighbouring countries were also discussed. Minutes of this meeting were circulated to the invited participants. 


\title{
Executive Committee Working Group: Encouraging the International Development of Antarctic Astronomy
}

\author{
Prepared by Michael Burton, Chairman
}

The session was held Wednesday, August 24, The Hague, Holland

the Opening Remarks were made by M. Burton (Chair, Australia). Then the following presentations were made:

Determination of Stellar Coordinates using Observations from Antarctica: G. Pinigin (Ukraine) \& Millimeter Cosmology: Jeff Peterson Carnegie-Mellon, USA

Several sensitive searches for anisotropy in the $2.7, \mathrm{~K}$ cosmic background radiation have been carried out at the South Pole in recent years, and several more are planned for the next few years. These observations will be described and the advantages and limitations of the South Pole site will be discussed.

Site Testing at the South Pole: Michael Burton, University of New South Wales, Australia

The combination of cold, dry, tenuous and stable air above the Antarctic Plateau provides unique conditions on the surface of the Earth for astronomical observations, particularly in the infrared and submillimetre wavebands. While these facets of the Antarctic environment have been long recognized, little has been done till recently to quantify these site conditions of the Plateau, as they affect the conduct of observational astronomy.

The University of New South Wales is now operating two site testing experiments at the South Pole, in collaboration with the Center for Astrophysical Research in Antarctica and the Universite de Nice.

The first experiment uses a single-element near-infrared photometer to make measurements of the sky brightness in the 1-5 microns band, and in particular to measure the sky spectrum with $1 \backslash \%$ resolution. It is particularly concerned with quantifying how low a level the night time sky emission drops to between 2.27 and 2.45 microns, where the airglow lines are absent and the thermal emission from sky and telescope is vastly reduced from mid-latitude sites.

The second experiment measures the micro-turbulence in the surface layer above the ice through the micro-changes in the resistance of a thin filament, caused by small fluctuations in the air temperature. The experiment is measuring the contribution of this turbulence to the site seeing at the South Pole at three levels, 7-m, 17-m and 27-m, up a mast.

Some results from these experiments will be presented.

The Antarctic Submillimeter-wave Telescope and Remote Observatory (AST/RO) Project: A. Stark, Smithsonian Astrophysical Observatory, USA

AST/RO is a 1.7 meter diameter submillimeter-wave radio telescope scheduled to begin operations at the Amundsen-Scott South Pole station in December 1994. Site testing shows that the South Pole has exceptional submillimeter-wave sky transparency and stability. Trial observations have been carried out at Boston University over the past two years; the AST/RO is fully operational. A $230 \mathrm{GHz}$ SIS receiver and a $\mathrm{U}$. of Cologne acousto- optical spectrometer with 2048 channels in a $1.2 \mathrm{GHz}$ bandwidth have been used to collect several hundred test spectra, many of which are essentially 
perfect within the noise. A University of Illinois $492 \mathrm{GHz}$ SIS receiver with $160 \mathrm{~K}$ DSB noise temperature and a Radiometer Physics $\mathrm{GmbH} 492 \mathrm{GHz}$ Schottky-barrier diode receiver have also been installed and partially tested.

Absolute pointing is 10 arcsec rms. Scans of the sun show that the offset optics provide a gaussian diffraction-limited beam.

Research supported by CARA and the NSF under Cooperative Agreement No. DPP8920223

An Astronomical Observing Site at 78 Degrees South - The Astroantar Project (IATE, OAC. IAA), Mirta Moscon and Pablo Recabarren, Observatorio Astronomico de Cordoba, Argentina

This project of astronomical observations has developed at the Antarctic Base Belgrano since 1989. Base Belgrano is located at $78 \mathrm{~S}$ and $34 \mathrm{~W}$, and is active during all the year. The different projects carried on are: seeing measurements, determination of extinction coefficients and meteorological conditions, acquisition of data of medium and short period variable stars and RCoronae Borealis type, and $\mathrm{H}$-alpha solar observations. We use a CG11 Celestron Reflector Telescope, with a ST6 CCD Camera, run from a PC, with $\mathrm{U}, \mathrm{V}, \mathrm{B}, \mathrm{R}, \mathrm{I}$ and $\mathrm{H}$-alpha filters. Previous statistics show that there are several periods of one or two weeks of good atmospherical conditions and good seeing. We are also working on the determination of the influence of australis aurorae and ozone layer density variations on the astronomical data.

The LABEL (LABoratorio BELgrano) is the ionospherical observatory of the Base and gives the technical and logistic support to the astronomical station. An engineer, two technicians and an astronomical observer work there during the entire year, and data are processed in Cordoba. The Wedell Sea was unusually frozen in January this year, and the Admiral Irizar Argentine Icebreaker could not reach Base Belgrano. So, the project has been interrupted until next December.

IATE, OAC: Investigaciones en Astronomia Teorica y Experimental, Observatorio Astronomico de Cordoba, Universidad Nacional de Cordoba. Argentina.

CONICET: Consejo de Investigaciones Cientificas y Tecnologicas.

IAA: Instituto Antartico Argentino.

Automated Astrophysical Observatories for the High Plateau: John Storey, University of New South Wales, Australia\}

Preliminary measurements of conditions at the South Pole confirm the expectation that inland sites in Antarctica can provide the best observing conditions on the surface of the earth for certain types of astronomy. It is now important that we determine which of the possible Antarctic sites is the best, and how much better that site is than other, possibly more accessible sites. A site testing program has therefore been put in place to study conditions at sites remote from human habitation. This program will be carried out as a collaboration between the Australian organisation JACARA and the US CARA.

The site testing will be carried out using an Automated Geophysical Observatory (AGO), in which low-power, automated astronomical instruments have been installed. Built by Lockheed under NSF funding, the AGO is an autonomous, self-heated, selfpowered laboratory which is deployed by Hercules aircraft and can run unattended for up to a year. Data are recorded on optical disc and recovered at the end of the twelve-month period. The first astronomically instrumented AGO is scheduled for initial testing at the South Pole in 1996, prior to deployment at a remote site. 
Russian plans for Antarctic Astronomy: Kirill Maslennikov, The Central Astronomical Observatory, Russian Academy of Sciences

The Russian science community (and astronomers in particular) have far-reaching intentions for developing activities in Antartica. In spite of the present crisis state of Russian economy it is hardly possible that the vast totality of experience accumulated by Russian investigators in Antarctica could be lost without use.

The present activities of Russian astronomers in the area of Antarctic astronomy are concentrated under the coordination of The Astronomical Society of Russia. In its frame the working group was formed in 1991 (chairman: Dr. V.Burdyuzha). The most involved institutes are:

The Astro-Space Center, Lebedev Physical Institute, Moscow; The Space Research Institute, Moscow;

The Central Astronomical Observatory, Pulkovo, St.Petersburg;

The Arctic and Antarctic Institute, St.Petersburg.

Here is a brief summary of the activities in this field:

The preliminary project of the International Antarctic Observatory was developed (and reported by Dr.Burdyuzha at IAU General Assembly in Buenos-Aires). Now the further development of the project is to be performed involving military industrial enterprises which are converted to the scientific and civil production. litem The Soviet Antarctic Vostok base supplied the vast body of climate and meteorology data for more than 30 years. Particularly the temperature distributions with height were being obtained almost daily thus defining the structure function and seeing parameters. These data are now under processing. The Vostok base is now conservated but is being restored to use in a few months. Its position seems very accessible due to the height $(3480 \mathrm{~m})$ and proximity to Dome Al@. Another basepoint, Komsomolskaya, might be even more promising. There are now three instruments being modified and winterised for operations in Antarctica:

IR hygrometer tested at Vostok base and destined to site testing; Submillimeter photometer based on the one used at Pamir base of Pulkovo Observatory at $4300 \backslash, m$ above the sea level under severe winter conditions;

IR photometer/polarimeter (0.3--3 microns) designed at Pulkovo Observatory.

Communications Needs for Antarctic Astronomy: Bob Loewenstein, Center for Astrophysical Research in Antarctica, USA

As large scale Antarctic astronomical projects such as the Center for Astrophysical Research in Antarctica (CARA) and the Antarctic Muon and Neutrino Detection Apparatus (AMANDA) become operational, it is estimated that data throughputs on the order of a Gigabyte/day and better interactive communication with winter-over personnel will be required.

In recent years the Internet was not available to the Amundsen-Scott South Pole Station; communication consisted of low bandwidth (1200-2400 bits/sec) transmission over two aging US Satellites (LES 9 and ATS 3), visible at the pole for a total of 9 hours. Communication sessions were non-interactive and typical data throughputs were 2 Mbytes per day.

First attempts to satisfy these communications needs occurred during the 1993-94 summer season at the South Pole Station. The first usable Internet connection to the South Pole was made in February 1994. The connection continues through this south 
polar winter using two satellites (GOES 2 and LES 9) with current data rates of 24 $\mathrm{Kbits} / \mathrm{sec}$ for a total of 9 hours per day and an effective throughput of about 30 Mbytes/day. It is possible to achieve rates up to $512 \mathrm{Kbits} / \mathrm{sec}$ on GOES 2 for 3 hours/day and $128 \mathrm{Kbits} / \mathrm{sec}$ on LES 9 for 6 hours/day, yielding a total daily theoretical throughput of about 100 Mbytes/day, far short of estimated needs.

The most viable means of providing the necessary bandwidths to the South Polar region is to continue to identify and acquire usage of several existing satellites that have outlived their original purposes. Other possible methods will be discussed.

The International Antarctic Balloon Observatory: Michael Dopita, Mount Stromlo and Siding Spring Observatories, Australia

Assuming a successful Australian/US program of site testing at the South Pole and at Dome C (Circe/Concorde), we have proposed the construction, as an international partnership project, of an International Antarctic Balloon Observatory (IABO), to be deployed above the Antarctic high plateau at an altitude of $8-10 \mathrm{~km}$. This observatory facility would be of the $2.5 \mathrm{~m}$-class, and would be designed to provide unique imaging capabilities in the visible and near IR (the POST concept), or to provide unequalled sensitivity in the far-IR. The considerations relating to the choice of observing site are discussed, and some aspects of the IR mission are emphasised.

Determination of Stellar Coordinates using Observations from Antarctica: G.I. Pinigin, Nikolaev Astronomical Observatory, Ukraine\}

There is intense interest amongst the astronomical community to the selection of the best sites on the Earth for making more precise and potential observations of celestial bodies. The XXI IAU General Assembly approved the project of the international Antarctic astronomical observatory (AAO) and resolved to create a working group "encouraging international development of Antarctic astronomy", headed by Dr. P. Gillingham. This project provides for the best observing conditions on the Earth in the infrared, sub-mm and $\mathrm{mm}$ wavelength range, a very dry, cold and tenuous atmosphere. It plans to establish the international AAO on the high Antarctic Plateau with the latitude about 89-90 degrees. This observatory will be an intermediate base before the future astronomical observatory on the Moon. 